\title{
Verbal or Visual Memory Score and Regional Cerebral Blood Flow in Alzheimer Disease
}

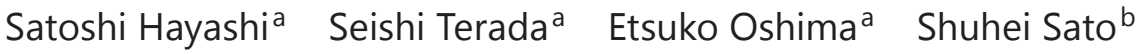 \\ Kairi Kurisu $^{a}$ Shintaro Takenoshita ${ }^{a}$ Osamu Yokota ${ }^{a}$ Norihito Yamada ${ }^{a}$ \\ a Department of Neuropsychiatry, Okayama University Graduate School of Medicine, \\ Dentistry and Pharmaceutical Sciences, Okayama, Japan; ${ }^{b}$ Department of Health \\ Information, Kawasaki University of Medical Welfare, Kurashiki, Japan
}

\section{Keywords}

Alzheimer disease $\cdot$ Cerebral blood flow $\cdot$ Memory $\cdot$ Verbal memory $\cdot$ Visual memory

\section{Abstract}

Objective: Among many cognitive function deficits, memory impairment is an initial and cardinal symptom in Alzheimer disease (AD). In most cases, verbal and visual memory scores correlate highly, but in some cases the deficit of verbal or visual memory is very different from that of the other memory. In this study, we examined the neural substrates of verbal and visual memory in patients with AD. Methods: One hundred eighty-eight consecutive patients with $A D$ were recruited from outpatient units. Verbal and visual memory scores were evaluated using the Wechsler Memory Scale - revised. The patients underwent brain SPECT with

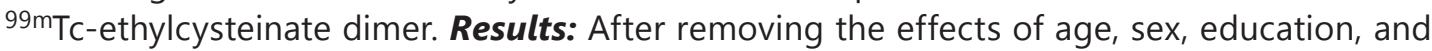
Mini-Mental State Examination scores, correlation analysis showed a significant correlation of verbal memory scores to regional cerebral blood flow ( $\mathrm{rCBF}$ ) in the bilateral cingulate gyrus and left precuneus. Similarly, a significant correlation of visual memory scores to rCBF was found in the right precuneus and right cingulate gyrus. Conclusion: The posterior medial cortices (PMC) are very important areas in episodic memory among patients with mild AD. Verbal memory is more closely related to the both sides of the PMC, while visual memory is more closely related to the right PMC. 
Hayashi et al.: Verbal or Visual Memory and rCBF in AD

\section{Introduction}

Alzheimer disease (AD) is the leading cause of late-onset dementia worldwide. A progressive decline in many brain functions is typical of AD, and among many cognitive functions, memory impairment is an initial and cardinal symptom in most patients with AD [1]. Uneven impairment across memory systems has been documented in mild AD [2]. Episodic memory is generally the first and most severely affected, while semantic memory is more resistant to loss $[1,2]$.

To detect the early deterioration of episodic memory among amnestic patients, the Wechsler Memory Scale - revised (WMS-R) is often used at many memory clinics in Japan. In the WMS-R, three major indices are obtained, namely, indices of general memory, attention, and delayed memory $[3,4]$. The general memory index consists of verbal and visual memory indices. Both verbal and visual memory indices reflect immediate episodic memory. In most cases with amnesia, verbal and visual memory indices correlate highly, but in some cases the severity of verbal or visual memory is very different from that of the other memory.

Numerous studies have investigated the neural substrate of verbal memory scores in patients with $\mathrm{AD}[1,2,5-11]$. Regional cerebral blood flow ( $\mathrm{rCBF}$ ) in many cortical areas was reported to be significantly correlated with verbal memory scores in AD patients. Meanwhile, there are fewer reports on the relationship of the visual memory score to $\operatorname{rCBF}[1,2,12,13]$. Some showed that $\mathrm{rCBF}$ in the posterior medial cortices (PMC) was correlated with visual memory scores $[2,13]$. It is asserted that global cognitive impairment should be taken into account when the correlation between $\mathrm{rCBF}$ and the neuropsychology score is investigated [6]. However, in most previous studies, no correction for general cognitive function was included in the analysis. Moreover, few studies compared the neural substrates of verbal and visual memory scores. Therefore, in this study, we compared the neural substrates of verbal and visual memory in patients with $\mathrm{AD}$ when corrected for global cognitive impairment.

In $\mathrm{AD}$ subjects, low scores on the word list learning test and delayed recall test were associated with left dominant hypoperfusion/hypometabolism in the PMC after correction for general cognitive function $[6,9,11]$. However, there have been no studies of visual memory controlling for the effect of general cognitive function. In this study, we hypothesized that the medial posterior cortex was the significant area, and that right predominance in visual memory and left predominance in verbal memory might be observed.

\section{Methods}

\section{Subjects}

This is a retrospective study. One hundred eighty-eight consecutive patients with AD who had visited the outpatient units of the Memory Clinic of Okayama University Hospital between January 2006 and December 2011 were recruited according to the following criteria.

They all (i) underwent general physical and neurological examinations and extensive laboratory testing, including thyroid function tests, serum vitamin $\mathrm{B}_{12}$, and syphilis serology; (ii) took the Mini-Mental State Examination (MMSE) [14] and the WMS-R [3, 4]; (iii) underwent single photon emission computed tomography (SPECT) with ${ }^{99 \mathrm{~m}}$ Tc-ethylcysteinate dimer of the brain as well as magnetic resonance imaging (MRI) or computed tomography (CT) of the head; and (iv) were diagnosed with probable AD according to the criteria formulated by the NINCDS-ADRDA [15]. The exclusion criteria were (i) complications from other neurological diseases or illnesses; (ii) history of mental illness or substance abuse prior to the onset of dementia; (iii) evidence of focal brain lesions on head MRI or head CT; (iv) treatment with antipsychotics, antidepressants, or anxiolytic drugs; (v) recent changes in medication likely to affect brain perfusion; and (vi) left handedness or ambidexterity. The profile of each subject (age, sex, months of disease duration, and years of education) was obtained by the chief clinician. 


\section{Instruments}

The WMS-R is the most widely used measure of adult memory in Japan [3, 4]. In the WMS-R, verbal and visual memory indices are obtained. Both verbal and visual memory indices evaluate immediate episodic memory. The mean value of both scores is 100 , and a higher score indicates a better performance. The scores of both memory indices are already age-adjusted when the age of the subject is under 75 years. However, when the age of the subject is over 75, there are no age-adjusted scores of memory indices of the WMS-R in Japan. Moreover, indices of the WMS-R have no exact scores if the subject takes scores under 50 . Therefore, in this study, instead of verbal and visual indices, we used verbal and visual scores. Verbal and visual scores are raw scores before age adjustment. Both scores are obtained as follows: verbal score $=$ logical memory $\mathrm{I} \times 2+$ verbal paired associates I, and visual score $=$ figural memory + visual paired associates + visual reproduction.

Ethics

This study was approved by the Internal Ethical Committee of Okayama University Graduate School of Medicine, Dentistry and Pharmaceutical Sciences. After a complete description of the study to the subjects and their relatives, written informed consent was obtained.

\section{Brain Perfusion SPECT Imaging}

All subjects were examined by brain perfusion SPECT. Patients were examined in a comfortable supine position with their eyes closed in quiet surroundings. Ten minutes after intravenous administration of ${ }^{99 \mathrm{~m}} \mathrm{Tc}-$ ethylcysteinate dimer (600 MBq; FUJIFILM RI Pharma Co., Ltd., Tokyo, Japan), SPECT images were obtained using a triple-head, rotating gamma camera interfaced with a minicomputer (GCA9300A/DI; Toshiba, Tokyo, Japan) equipped with a fanbeam, low-energy, high-resolution collimator. Sixty projection images over a $360^{\circ}$ angle in a $128 \times 128$ matrix were acquired. All images were reconstructed using ramp-filtered back projection and then smoothed three-dimensionally with a Butterworth filter (order 8, cutoff $0.12 \mathrm{cycles} / \mathrm{cm}$ ). The reconstructed images were corrected for gamma-ray attenuation using the Chang method $(\mu=0.09)[16]$.

\section{Data Analysis}

Spatial preprocessing and statistical analysis of images were performed on a voxel-by-voxel basis using Statistical Parametric Mapping 8 (SPM8; Wellcome Department of Imaging Neuroscience, UK) running on MATLAB (MathWorks, Inc., Natick, MA, USA). All SPECT images of each subject were normalized to the standard brain of the Montreal Neurological Institute (MNI), and spatial normalization was performed with 12-parameter affine and nonlinear transformations [17]. The voxel sizes of the reslice option were $2 \times 2 \times 2$ $\mathrm{mm}$. The nonlinear parameters were set at $25 \mathrm{~mm}$ cutoff basis functions and 16 iterations. All the normalized SPECT images were then smoothed with an isotropic gaussian kernel filter $(12 \mathrm{~mm}$ full-width at halfmaximum).

We applied a simple regression method using SPM8 to obtain the correlation between WMS-R verbal or visual scores and rCBF imaging data from SPECT of the 188 AD subjects with age, sex, and education entered into the model as nuisance covariates. Thereafter, to remove the effect of general cognitive function, total MMSE scores were entered into the model as nuisance covariates in addition to age, sex, and education. Thereafter, we performed a simple regression method using SPM8 to obtain the correlation between WMS-R verbal or visual scores and rCBF imaging data from SPECT.

The specific effects of WMS-R scores were tested [1] using t-contrast with an additional zero for the scores of other factors, assuming that the extent of the symptoms would be uniquely associated with decreased rCBF. In both analyses, a threshold of $p<0.05$ (corrected, family-wise error) was used at the voxel level, and results were considered significant at 50 voxels at the cluster level. In both analyses, global normalization was performed by proportional scaling with the mean voxel value. Masking was applied using the threshold method (0.8 times the global value). Other statistical analyses were performed using the SPSS 14.0 J software program (SPSS Inc., Chicago, IL, USA).

\section{Results}

Clinical Characteristics and Neuropsychological Tests

Among the 188 AD patients, 121 were women and 67 were men. For dementia severity, 116 patients had clinical dementia rating (CDR) scores of $0.5,66$ had CDR of 1 , and 6 had CDR 
Table 1. Clinical characteristics of patients $(n=188)$

\begin{tabular}{lcrr}
\hline & Mean & SD & \multicolumn{1}{c}{ Range } \\
& & & \\
\hline Age, years & 74.8 & 7.7 & $48-89$ \\
Disease duration, months & 31.8 & 20.1 & $4-84$ \\
Education, years & 11.3 & 2.5 & $6-18$ \\
MMSE (0-30) & 22.1 & 3.0 & $16-29$ \\
WMS-R & & & \\
$\quad$ Verbal memory & 19.3 & 9.6 & $1-47$ \\
$\quad$ Visual memory & 31.3 & 10.6 & $6-59$ \\
\hline
\end{tabular}

MMSE, Mini-Mental State Examination; WMS-R, Wechsler Memory Scale - Revised; SD, standard deviation.

of 2. Other demographic characteristics are shown in Table 1. Pearson's correlation coefficients were 0.375 between MMSE and WMS-R verbal memory scores, 0.386 between MMSE and WMS-R visual memory scores, and 0.487 between WMS-R verbal and visual memory scores.

\section{Verbal Memory Scores and rCBF}

Figure $1 \mathrm{a}$, and $\mathrm{b}$, and Table 2 show the SPM ( $\mathrm{t}$ ) map of significant correlations between rCBF and WMS-R verbal memory scores among AD patients. Correlation analysis after removing the effects of age, sex, and education showed a significant cluster of voxels in the left precuneus and left cingulate gyrus (Brodmann areas 7 and 31) (Fig. 1a). After removing the effects of MMSE scores in addition to age, sex, and education, correlation analysis showed a significant cluster of voxels in the bilateral cingulate gyri and left precuneus (Brodmann areas 7 and 31) (Fig. 1b). Table 2 shows the probability results of the SPM analysis and the location of peak Z-scores in terms of MNI coordinates.

\section{Visual Memory Scores and $r C B F$}

Figure 1c, and d, and Table 3 show the SPM (t) map of significant correlation between rCBF and WMS-R visual memory scores among AD patients. Correlation analysis after removing the effects of age, sex, and education showed a significant cluster of voxels in the right precuneus and right cingulate gyrus (Brodmann areas 7 and 31) (Fig. 1c). After removing the effects of MMSE scores in addition to age, sex, and education, correlation analysis again showed a significant cluster of voxels in the right precuneus and right cingulate gyrus (Brodmann areas 7 and 31) (Fig. 1d). Table 3 shows the probability results of the SPM analysis and the location of peak z-scores in terms of MNI coordinates.

\section{Discussion}

There have been many studies on the relationship of cerebral regions to verbal memory scores in AD (Table 4). Verbal memory scores are reported to be significantly correlated with the rCBF in almost all cortical areas in at least one study. However, various psychological tests were used to evaluate verbal memory. Therefore, the differences in the results were somewhat dependent on methodological differences. The areas of $\mathrm{rCBF}$ that were frequently reported to be significantly correlated with verbal memory scores (at least 4 times in the 16 analyses excluding our own) were the left posterior cingulate gyrus, bilateral precuneus, and left middle temporal gyrus. 

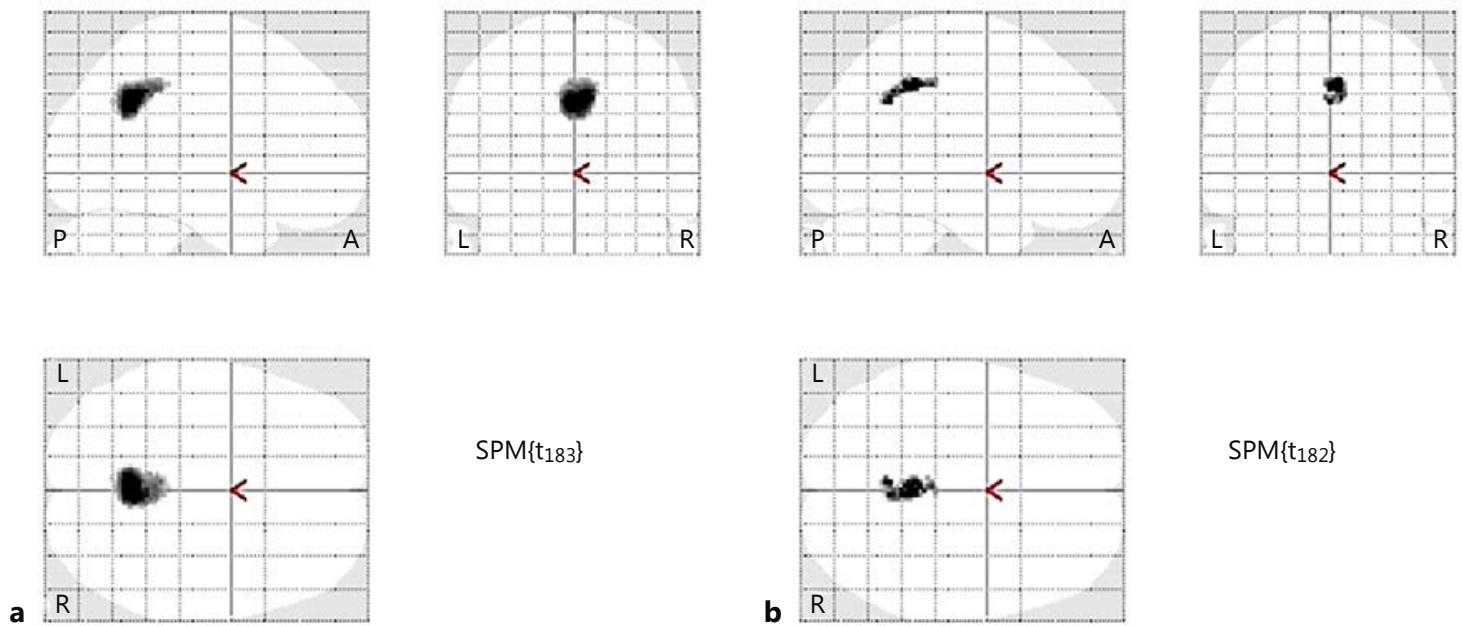

$\mathrm{SPM}\left\{\mathrm{t}_{183}\right\}$
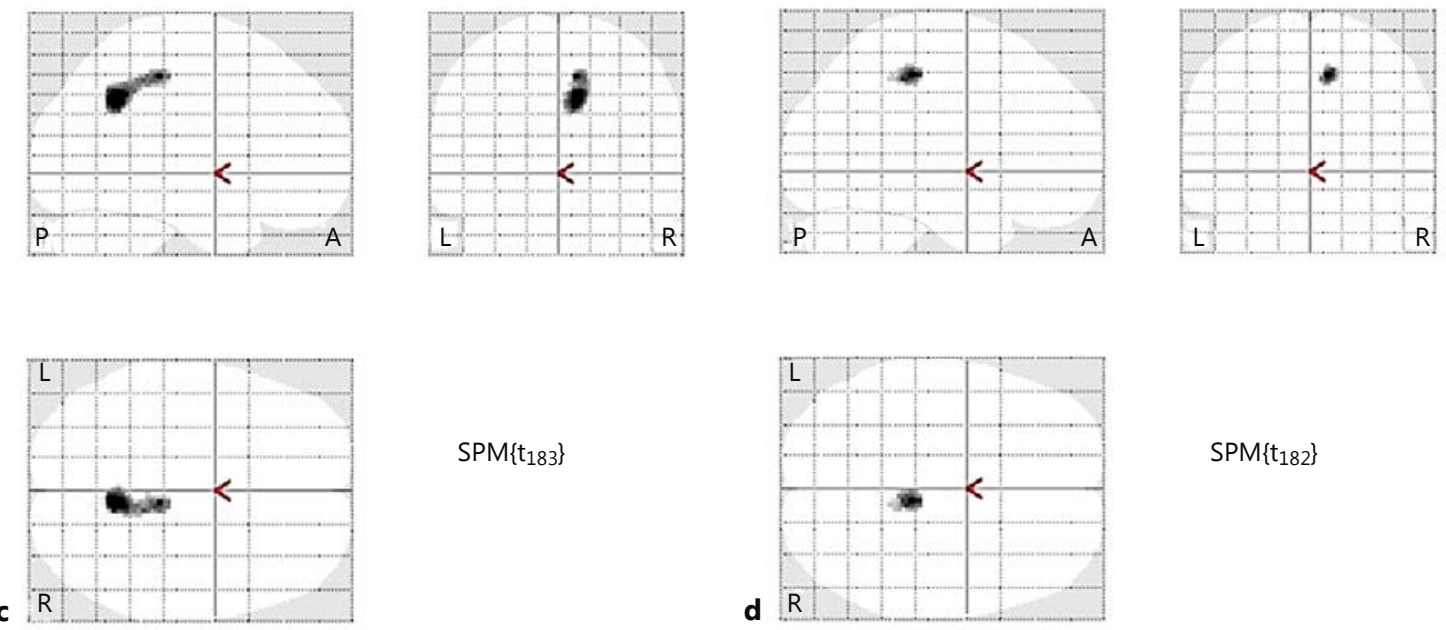

$\operatorname{SPM}\left\{\mathrm{t}_{183}\right\}$

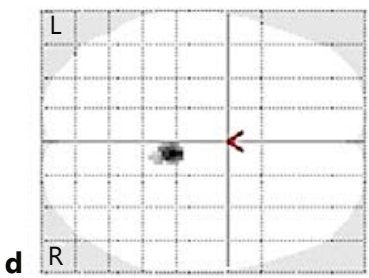

$\operatorname{SPM}\left\{\mathrm{t}_{182}\right\}$

Fig. 1. a The Statistical Parametric Mapping (SPM) (t) map of significant correlations between regional cerebral blood flow ( $\mathrm{rCBF}$ ) and verbal memory scores among Alzheimer disease (AD) patients after removing the effects of age, sex, and education. $\mathbf{b}$ The SPM ( $\mathrm{t}$ ) map of significant correlations between rCBF and verbal memory scores among AD patients after removing the effects of age, sex, education, and MMSE scores. c The SPM $(\mathrm{t})$ map of significant correlations between $\mathrm{rCBF}$ and visual memory scores among AD patients after removing the effects of age, sex, and education. $\mathbf{d}$ The SPM $(\mathrm{t})$ map of significant correlations between rCBF and visual memory scores among AD patients after removing the effects of age, sex, education, and MMSE scores. a-d Three-way glass view of the area of significant correlation. Upper right, coronal; upper left, sagittal; lower, transverse.

Verbal memory is usually an early deficit in AD, and its impairment largely reflects the severity of the disease in the early stage [6]. Therefore, if the severity of AD is not taken into account, it is highly possible that the correlation always reflects the sites of perfusion/metabolism impairment in early AD rather than the sites of verbal memory. In a study that corrected for the general cognitive function with a strict significance level, the rCBF in the bilateral precuneus (Brodmann areas 7 and 31) and left parietal lobule was significantly related to verbal memory scores [11]. 
Dementia

Cognitive Disorders

Hayashi et al.: Verbal or Visual Memory and rCBF in AD
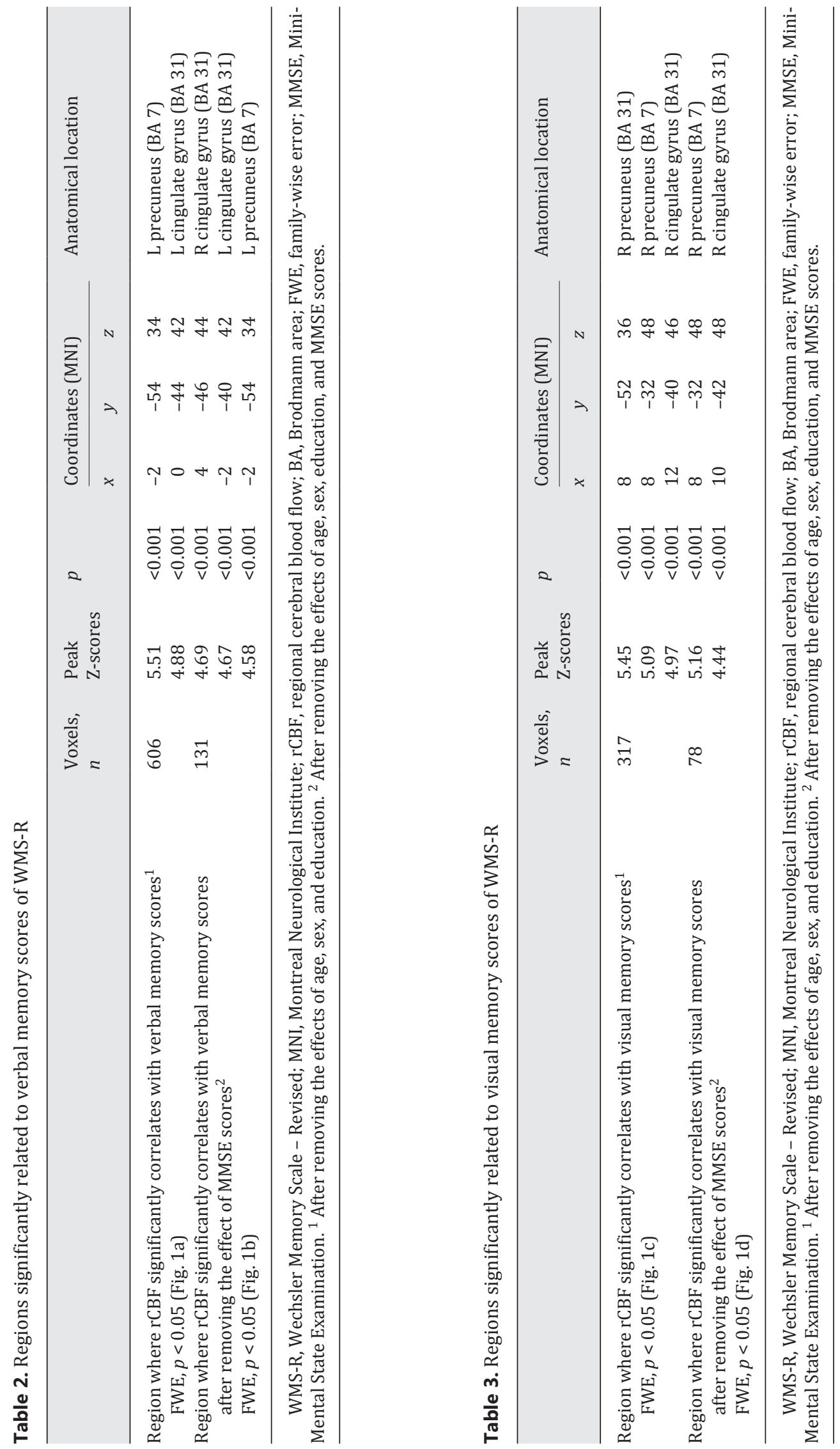
Dementia

Cognitive Disorders

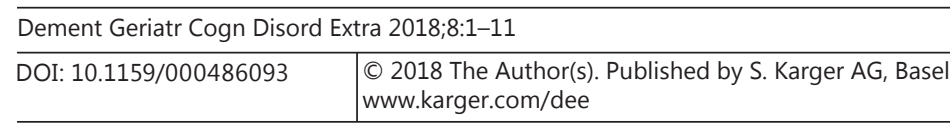

Hayashi et al.: Verbal or Visual Memory and rCBF in AD

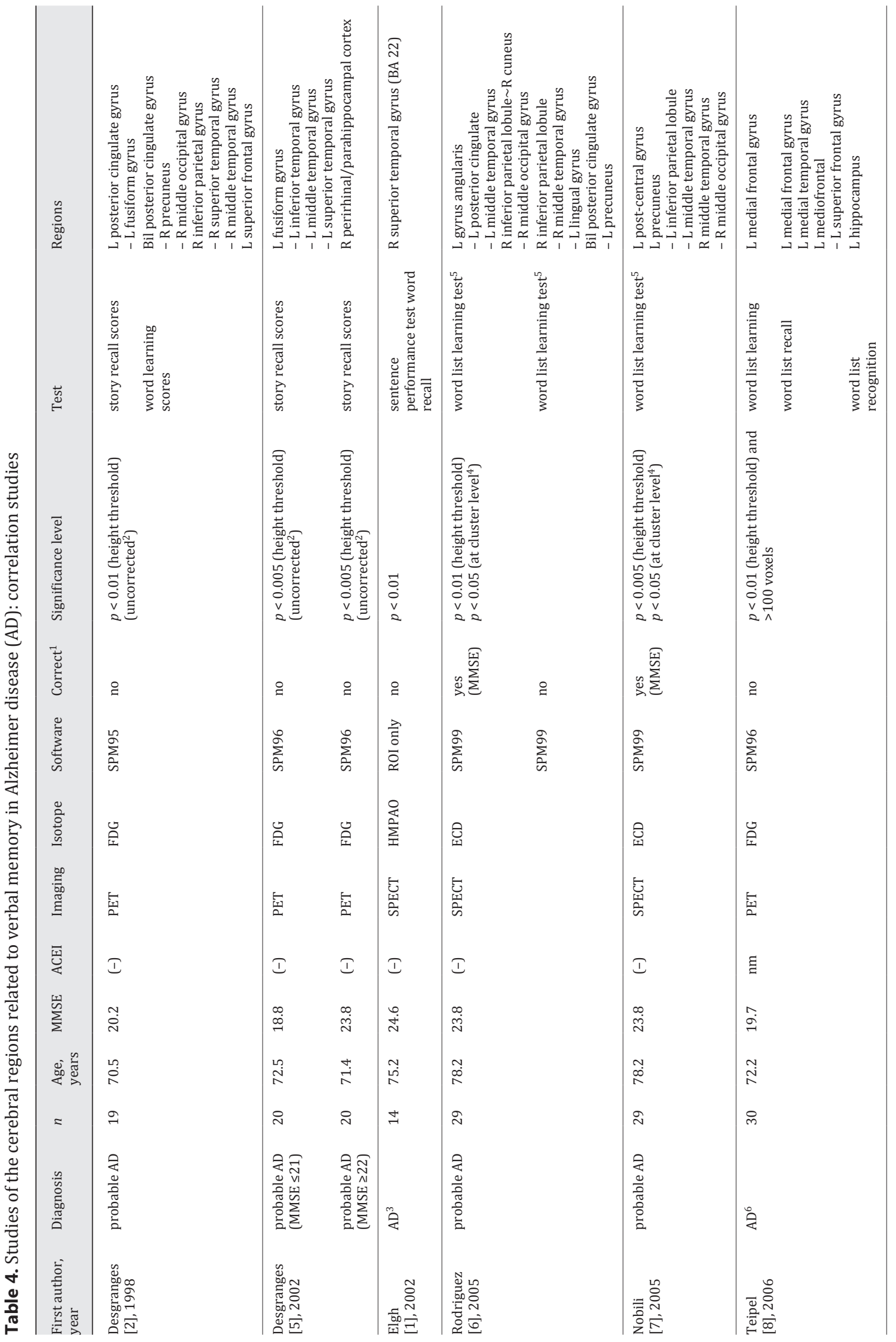


Dementia

Cognitive Disorders

Hayashi et al.: Verbal or Visual Memory and rCBF in AD

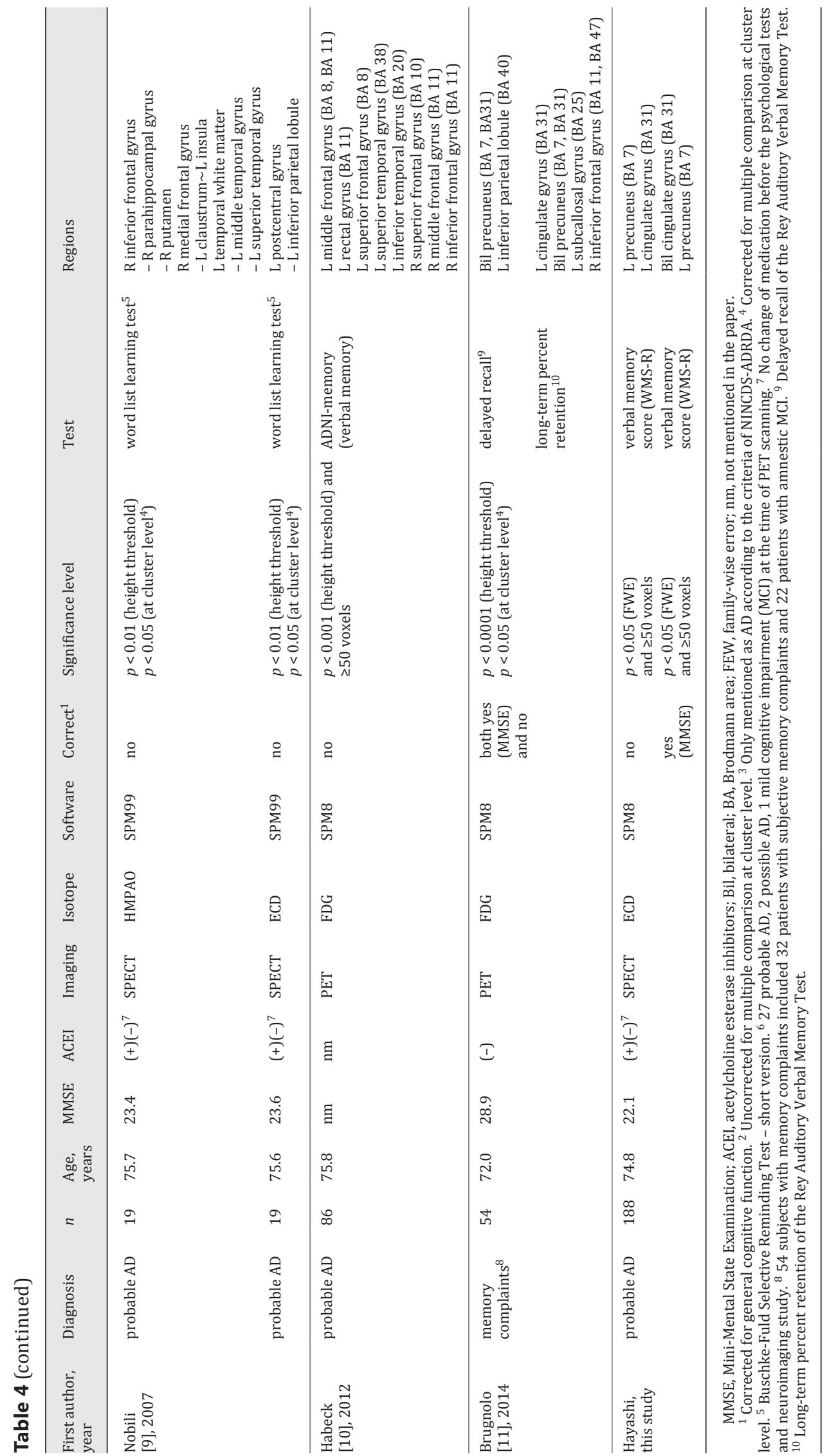


Dxina

Dementia

Cognitive Disorders

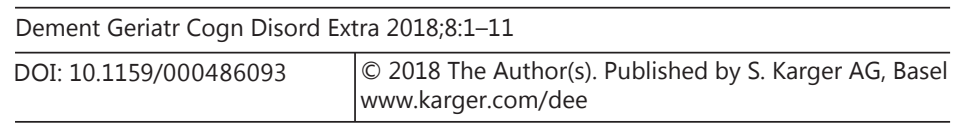

Hayashi et al.: Verbal or Visual Memory and rCBF in AD

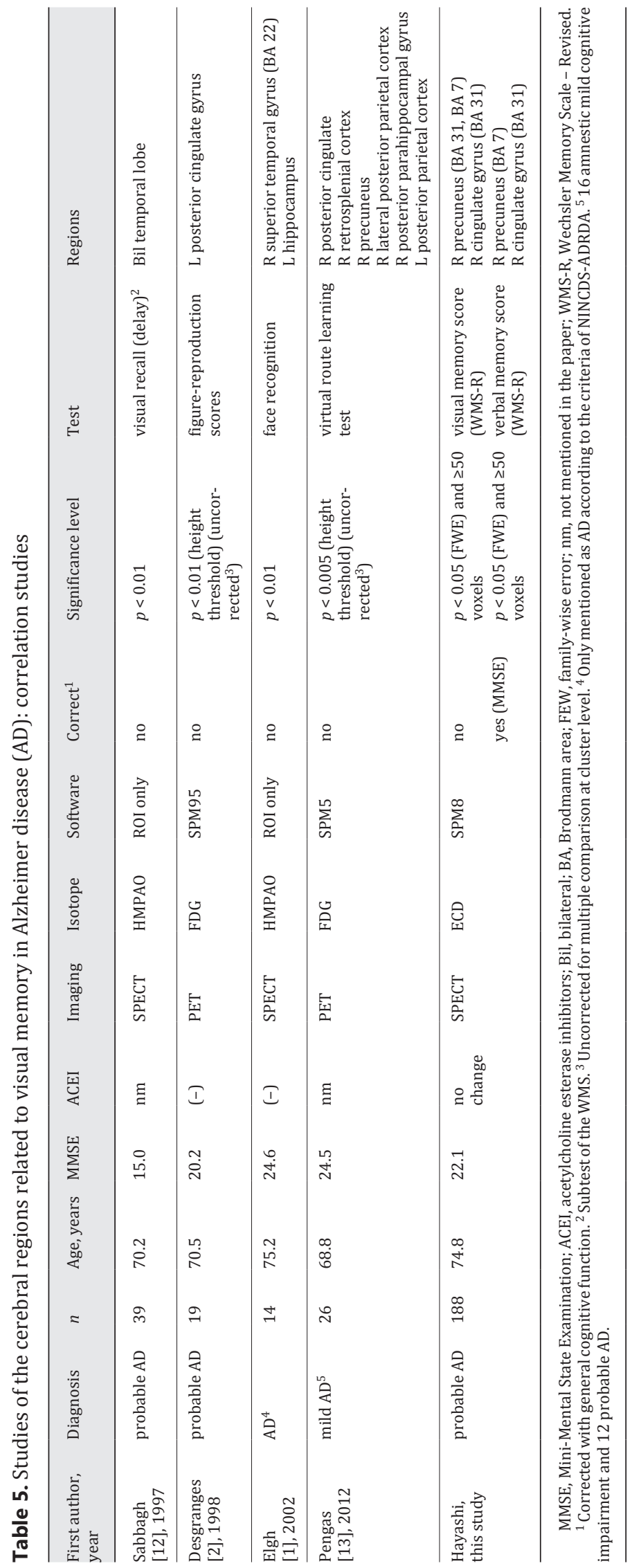


In this study, verbal memory scores correlated with $\mathrm{rCBF}$ in the left precuneus and bilateral posterior cingulate gyrus (Brodmann areas 7 and 31) in both situations with and without correction for general cognitive function. Participants in this study were mostly patients with mild dementia, and the difference in general cognitive function among participants in this study is relatively limited. Therefore, we think that correlation both before and after correction for general cognitive function showed similar results. Moreover, the significance level of correlation of rCBF in this study is stricter than those in most other studies (Table 4). The stricter significance level might make the more specific narrow area significant.

There have been few studies on the relationship of the visual memory score to hypoperfusion/hypometabolism compared to verbal memory score (Table 5). There are conflicting views on the laterality of visual memory scores. One study reported right predominance [13] and the other left predominance [2]. However, there have been no studies controlling for the effect of general cognitive deterioration. We found that $\mathrm{rCBF}$ in the right PMC was significantly correlated with visual memory scores in both situations with and without correction for general cognitive function. Thus, we think that the right PMC plays an important role in visual memory tasks.

The role of the PMC in memory processing has been confirmed for structures involved in the default mode network, namely the posterior cingulate and precuneus [18, 19]. Recent functional imaging findings in healthy subjects suggest a central role for the precuneus and posterior cingulate in a wide spectrum of highly integrated tasks, including episodic memory retrieval [18]. Memory functions of the PMC have been studied mainly by fMRI [20, 21], and activation of the PMC was observed during episodic, but not semantic, memory recall tasks [22]. In studies using PET, paired word learning and cued word recall tests were reported to be associated with left dominant activation in the PMC $[23,24]$. On the other hand, the right PMC is closely involved in internally focused attention [25]. Memory performance associated with the posterior cingulate cortex (PCC) has been based on intrinsic hippocampal-PCC connectivity [26]. We also found that the PMC is a very important area in episodic memory in mild AD.

There are several limitations in this study. First, we used the WMS-R in this study to evaluate verbal and visual memory because the WMS-III and WMS-IV have not been translated into Japanese. In Japan, the WMS-R is commonly used as a short-term memory function test. Secondly, it is possible that we overlooked some significant areas due to the low resolution of SPECT. The area where rCBF was significantly correlated with WMS-R scores in this study is not the only important area in performance on the WMS-R. Thirdly, our study included only AD outpatients at a memory clinic. Therefore, the results of this study cannot be generalized easily to persons with normal cognitive function or patients with other diseases. Irrespective of the shortcomings stated above, this is the first study to compare the neural substrate of verbal and visual memory, after taking into account the general cognitive level.

\section{Acknowledgments}

We sincerely thank Ms. Horiuchi, Ms. Imai, Ms. Yabe, and Ms. Yifei Tang for their skillful assistance. This work was supported by grants from the Japanese Ministry of Education, Culture, Sports, Science and Technology (15K09831) and the Zikei Institute of Psychiatry.

\section{Disclosure Statement}

The authors have no conflicts of interest to declare. 
Hayashi et al.: Verbal or Visual Memory and rCBF in AD

\section{References}

1 Elgh E, Sundström T, Näsman B, et al: Memory functions and $\mathrm{rCBF}^{99 \mathrm{~m}} \mathrm{Tc}-\mathrm{HMPAO}$ SPET: developing diagnostics in Alzheimer's disease. Eur J Nucl Med Mol Imaging 2002;29:1140-1148.

-2 Desgranges B, Baron JC, de la Sayette V, et al: The neural substrates of memory systems impairment in Alzheimer's disease. A PET study of resting brain glucose utilization. Brain 1998;121(Pt 4):611-631.

3 Wechsler D: Wechsler Memory Scale Manual, Revised. San Antonio, Psychological Corporation, 1987.

4 Sugishita M: Japanese Wechsler Memory Scale-Revised (in Japanese). Tokyo, Nihon Bunka Kagakusha, 2001.

5 Desgranges B, Baron JC, Lalevée C, et al: The neural substrates of episodic memory impairment in Alzheimer's disease as revealed by FDG-PET: relationship to degree of deterioration. Brain 2002;125(Pt 5):1116-1124.

6 Rodriguez G, Morbelli S, Brugnolo A, et al: Global cognitive impairment should be taken into account in SPECTneuropsychology correlations: the example of verbal memory in very mild Alzheimer's disease. Eur J Nucl Med Mol Imaging 2005;32:1186-1192.

7 Nobili F, Brugnolo A, Calvini P, et al: Resting SPECT-neuropsychology correlation in very mild Alzheimer's disease. Clin Neurophysiol 2005;116:364-375.

-8 Teipel SJ, Willoch F, Ishii K, et al: Resting state glucose utilization and the CERAD cognitive battery in patients with Alzheimer's disease. Neurobiol Aging 2006;27:681-690.

$\checkmark 9$ Nobili F, Koulibaly PM, Rodriguez G, et al: ${ }^{99 \mathrm{~m}} \mathrm{Tc}-\mathrm{HMPAO}$ and ${ }^{99 \mathrm{~m}} \mathrm{Tc}-\mathrm{ECD}$ brain uptake correlates of verbal memory in Alzheimer's disease. Q J Nucl Med Mol Imaging 2007;51:357-363.

10 Habeck C, Risacher S, Lee GJ, et al: Relationship between baseline brain metabolism measured using $\left[{ }^{18} \mathrm{~F}\right] \mathrm{FDG}$ PET and memory and executive function in prodromal and early Alzheimer's disease. Brain Imaging Behav 2012;6:568-583.

11 Brugnolo A, Morbelli S, Arnaldi D, et al: Metabolic correlates of Rey auditory verbal learning test in elderly subjects with memory complaints. J Alzheimer Dis 2014;39:103-113.

12 Sabbagh MN, Lynn P, Jhingran S, et al: Correlations between SPECT regional cerebral blood flow and psychometric testing in patients with Alzheimer's disease. J Neuropsychiatry Clin Neurosci 1997;9:68-74.

13 Pengas G, Williams GB, Acosta-Cabronero J, et al: The relationship of topographical memory performance to regional neurodegeneration in Alzheimer's disease. Front Aging Neurosci 2012;4:17.

14 Folstein MF, Folstein SE, McHugh PR: "Mini-mental state." A practical method for grading the cognitive state of patients for the clinician. J Psychiatry Res 1975;12:189-198.

15 McKhann G, Drachman D, Folstein M, et al: Clinical diagnosis of Alzheimer's disease: report of the NINCDSADRDA Work Group under the auspices of Department of Health and Human Services Task Force on Alzheimer's Disease. Neurology 1984;34:939-944.

16 Sloboda RS: Vector implementation of Chang's attenuation correction method for single photon emission computed tomography. Med Phys 1987;14:1045-1047.

17 Friston KJ, Ashburner J, Poline JB, et al: Spatial registration and normalization of images. Hum Brain Mapp 1995;2:165-189.

18 Cavanna AE, Trimble MR: The precuneus: a review of its functional anatomy and behavioural correlates. Brain 2006;129(Pt 3):564-583.

19 Haramati S, Soroker N, Dudai Y, et al: The posterior parietal cortex in recognition memory: a neuropsychological study. Neuropsychologia 2008;46:1756-1766.

20 Golby AJ, Poldrack RA, Brewer JB, et al: Material-specific lateralization in the medial temporal lobe and prefrontal cortex during memory encoding. Brain 2001;124(Pt 9):1841-1854.

21 Bonelli SB, Powell RH, Yogarajah M, et al: Imaging memory in temporal lobe epilepsy: predicting the effects of temporal lobe resection. Brain 2010;133(Pt 5):1186-1199.

22 Fletcher PC, Frith CD, Baker SC, et al: The mind's eye - precuneus activation in memory-related imagery. Neuroimage 1995;2:195-200.

23 Bäckman L, Andersson JL, Nyberg L, et al: Brain regions associated with episodic retrieval in normal aging and Alzheimer's disease. Neurology 1999;52:1861-1870.

24 Krause BJ, Horwitz B, Taylor JG, et al: Network analysis in episodic encoding and retrieval of word-pair associates: a PET study. Eur J Neurosci 1999;11:3293-3301.

25 Chen QL, Xu T, Yang WJ, et al: Individual differences in verbal creative thinking are reflected in the precuneus. Neuropsychologia 2015;75:441-449.

-26 Wang L, Laviolette P, O’Keefe K, et al: Intrinsic connectivity between the hippocampus and posteromedial cortex predicts memory performance in cognitively intact older individuals. Neuroimage 2010;51:910-917. 